\title{
Características clínicas de los pacientes con LNH del Hospital Arnau de Vilanova de Valencia
}

\author{
E. MONZÓ CASTELLANO, M. MORALES SUÁREZ-VARELA*, J. MAYANS \\ FERRER, V. VILA LÓPEZ* \\ Servicio de Hematología del Hospital Arnau de Vilanova. Valencia. *Unidad de Salud \\ Pública, Higiene y Salud Ambiental .Universidad de Valencia. Unidad Clínico- \\ Epidemiológica del Hospital Universitario Dr. Peset. Valencia
}

\begin{abstract}
CLINICAL CHARACTERISTICS OF PATIENTS WITH NON-HODG KIN LYMPHOMA (NHL) IN ARNAU DE VILANOVA HOSPITAL. VALENCIA, SPAIN
\end{abstract}

\section{RESUMEN}

Objetivo: Conocer las características clínicas de los pacientes con linfoma no Hodgkin (LNH) en general y por subtipos histológicos atendiendo a la clasificación REAL.

Pacientes y métodos: Sobre 210 historias clínicas de pacientes con LNH, se seleccionaron 188 con informe anatomopatológico completo que utilizaban la clasificación REAL o si utilizaban otra clasificación se convirtió a ésta por un experto. Recogiéndose las características clínicas del paciente en el momento del diagnóstico con las que se relacionó.

Resultados: Se han encontrado diferencias significativas en la distribución por sexos en los linfomas de células del manto, con predominio de los varones $(\mathrm{p}=0,005)$.

Los pacientes más jóvenes se diagnosticaron de linfoma de Burkitt y Linfoma Linfoblástico B.

El estado del paciente mostró significación estadística $(\mathrm{p}=0,0001)$ con predominio de ECOG 0 y el estadio de los pacientes estudiados fue de $74,1 \%$ (estadios III/IV) frente a $25,9 \%$ (estadios I/II).

Conclusiones: Las diferentes características de los pacientes con LNH parece hacer necesaria la consideración de utilizar una sola clasificación y del diagnóstico por subtipos histológicos.

PALABRAS CLAVE: Linfoma no Hodgkin. Características clínicas. Clasificación REAL.

\begin{abstract}
Objective: To define the clinical characteristics of patients with nonHodgkin lymphoma (NHL) in general and by histological subtypes based on the REAL classification.

Patients and methods: From a total of 210 case histories of patients with NHL, 188 presenting a complete pathology report were selected. The latter was either based on the REAL classification or was converted to it by an expert. The clinical features of the patients at the time of diagnosis were also recorded.

Results: Significant differences were observed in the distribution by sex among mantle cell lymphomas, with a predominance among males $(p=0.005)$. The youngest patients were diagnosed of Burkitt lymphoma and $B$ lymphoblastic lymphoma. Patient status improved significantly $(p=0.0001)$, with a predominance of ECOG 0 , and the stage of the patients studied was $74.1 \%$ (stage III/IV) versus $25.9 \%$ (stage I/II).

Conclusions: The different characteristics of patients with NHL sug gest the need for a single classification with diagnosis by histological subtype.
\end{abstract}

KEY WORDS: Non-Hodgkin lymphoma. Clinical characteristics. REAL classification.

\section{INTRODUCCIÓN}

Los linfomas no hodgkinianos (LNH) constituyen un grupo heterogéneo de neoplasia cuya incidencia ha sufrido un rápido aumento en los últimas décadas en todo el mundo, sin que se haya producido ningún cambio en sus criterios de diagnóstico $(1,2,3)$.

Presentan una gran variabilidad de subtipos histológicos que dificultan el diagnóstico con implicaciones en la clínica y en la respuesta al tratamiento. Es por esto que uno de los pro- blemas de la patología tumoral linfoide, tanto para los expertos en el tema como para los patólogos en general, es la dificultad que existe en el reconocimiento del subtipo neoplásico teniendo en cuenta las distintas clasificaciones utilizadas en el momento actual (4). La importancia de una correcta clasificación anatomopatológica se muestra por las numerosas clasificaciones que intentan este objetivo como son la clasificación de Rappaport (5), Kiel (6-7), International Working Formula tion (IWF) (8) y REAL (9-10).

Junto a esto nos encontramos la aparente aparición de

Trabajo aceptado: 27 de Junio de 2001

Correspondencia: María Morales Suárez-Varela. Unidad de Salud Pública, Higiene y Salud Ambiental. Facultad de Farmacia. Universidad de Valencia. Avda. Vicente Andrés Estellés, s/n. 46100 Burjassot. Valencia. Tel/ Fax: 96 3864951. E-mail: maria.m.morales@uv.es - mosuva@san.gva.es 
entidades nuevas, todas ellas con diferente respuesta terapéutica, lo que dificulta enormemente la decisión clínica respecto a cual es el tratamiento más adecuado en cada caso.

En este sentido, una aproximación válida consiste en analizar las características de aquellos pacientes con LNH bajo una sola clasificación que nos permite una mejor valoración de cada entidad anatomopatológica y su relación con la presentación clínica de esta enfermedad.

En nuestro estudio intentamos conocer las características clínicas en el momento del diagnóstico de los pacientes con linfoma en nuestro hospital por subtipos diagnósticos anatomopatológicos según la clasificación REAL.

\section{PACIENTES Y MÉTODOS}

Se realiza un estudio retrospectivo, dónde se recogieron las diferentes características de los pacientes con LNH.

El marco del estudio esta constituido por toda la población atendida en el Hospital Arnau de Vilanova de Valencia, hospital de referencia del Área 5 de la Consellería de Sanitat y Consum de la Comunitat Valenciana, con una población atendida según el Servei Valencia de Salut, 1995 de 216.005 habitantes (107.125 hombres y 108.880 mujeres). Para ello se utilizaron como fuente de consulta los datos estadísticos suministrados por el Servicio de Hematología del Hospital y el examen de las historias clínicas del archivo correspondiente a este mismo servicio.

Se revisaron 210 historias clínicas de enfermos con diagnosticados de LNH incluidos durante el periodo de enero de 1978 a marzo del 2000. De ellos se seleccionaron 188 $(89,52 \%)$ historias clínicas, utilizando como criterio de inclusión que fuesen historias completas con material suficiente para reconsiderar el diagnóstico anatomopatológico de aquellas que no utilizaban la clasificación REAL y que nos permitiese su conversión a esta clasificación por un experto hematólogo asesorado por un anatomopatólogo, que ha supuesto la reclasificación de un $90 \%$ de los diagnósticos encontrados.

Como criterio de exclusión en el estudio de los 24 restantes han sido la falta de datos o por un tiempo de estancia en el hospital insuficiente para una valoración correcta de la enfermedad o ser pacientes atendidos pero no diagnosticados en este hospital.

Hemos encontrado 163 diagnósticos anatomopatológicos diferentes utilizados durante este periodo correspondientes a las distintas clasificaciones utilizadas. Concretamente se han encontrado diagnósticos pertenecientes a las clasificaciones IWF, REAL, Rappaport y Kiel, que finalmente se han homologado todas con la clasificación REAL, como hemos indicado anteriormente, ya que esta última ha tenido en muy poco tiempo una gran resonancia internacional y puede afirmarse que es la clasificación de referencia actual, al menos para los patólogos (11).

Se recogieron y analizaron los siguientes parámetros:

-Datos clínicos: edad, sexo, estado general (según escala ECOG), fecha de primera visita, presencia de síntomas B.

1. Subtipo histológico, clasificación utilizada, equivalencia con la REAL, fecha de diagnóstico.

\section{Datos bioquímicos: LDH}

3. Datos de extensión tumoral: localización extraganglionar, número de áreas extraganglionares afectadas, estadio (según el sistema de Ann-Arbor) (12,13).
Para el procesamiento de los datos se utilizó un ordenador personal, introduciendose los datos en base de datos Microsoft Excell 2000. El análisis estadístico se realizó con el paquete estadístico SPSS para windows 8.0.

\section{ANÁLISIS ESTADÍSTICO}

Para este análisis se realizó un estudio descriptivo de las variables utilizadas en el caso de tratarse de variables cualitativas, frecuencias absolutas, relativas e intervalo de confianza al 95\%. Las variables cuantitativas se expresaron como media más menos desviación estándar y las cualitativas como frecuencias o porcentajes.

Se utilizó la prueba ANOVA para la comparación de medias entre diagnósticos en los casos de número de áreas y LDH, y la prueba de Chi-cuadrado de Pearson siendo necesario en algunas ocasiones con la corrección de Yates para la comparación de proporciones, ambas con un nivel de confianza del $95 \%$ y sus equivalentes no paramétricos cuando fue preciso.

\section{RESULTADOS}

Los datos mostrados en la tabla I demuestran que no se han encontrado diferencias significativas en la distribución por sexos (101 varones y 85 mujeres. Razón de masculinidad 1,18). Esta misma situación de igualdad hombre:mujer se mantiene por subtipos histológicos según la clasificación REAL a excepción de los linfomas de células del manto en el que predominan los varones $(\mathrm{p}=0,005)$.

La edad media de la primera visita fue de 60,6 (DS: 15,6, rango de 15-93) años sin mostrar diferencias significativas con la edad del diagnóstico de 61,09 (DS: 14,76, rango de 1793). Sin embargo, los pacientes más jóvenes fueron diagnosticados de linfoma de Burkitt y de linfoma linfoblástico B. Los de más edad fueron diagnosticados de linfoma de células del manto, seguido del linfoma tipo Malt $(66,7 \%)$ y de linfoma centrofolicular difuso (56\%).

En cuanto a los valores de LDH y del estado del paciente en la primera visita (ECOG). El valor de la LDH no muestra diferencia estadísticamente significativa $(p=0,209)$ entre los diferentes subtipos histológicos que conforman la serie valorada conjuntamente, aunque comparados de forma bivariante si aparecen diferencias en los niveles de la LDH de los linfomas B de alto grado (LDH 1.300-1.500) y linfoma de bajo grado tipo tricoleucemia o inmunocitoma (LDH: 300). Sin embargo el estado del paciente si presenta una diferencia estadísticamente significativa $(\mathrm{p}=0,0001)$ respecto al estado general del paciente, con predominio del ECOG 0, es decir los pacientes son diagnosticados mayoritariamente con un estado de salud aparentemente bueno $(66,1 \%)$.

En el estadio de los pacientes estudiados de LNH, se observa que la mayoría se encuentra en el estadio IV $(57,3 \%)$. Esto supone un $74,1 \%$ en estadio III/IV frente a un $25,9 \%$ en estadio I/II.

En los casos en que existen diferencias significativas en los estadios I/II frente III/IV (linfoma difuso de células grandes, de células $\mathrm{T}$ periféricas, linfocítico de células pequeñas. linfoplasmocitoide, centrofolicular nodular y centrofolicular difuso) predomina el estadio IV (Tabla II). 


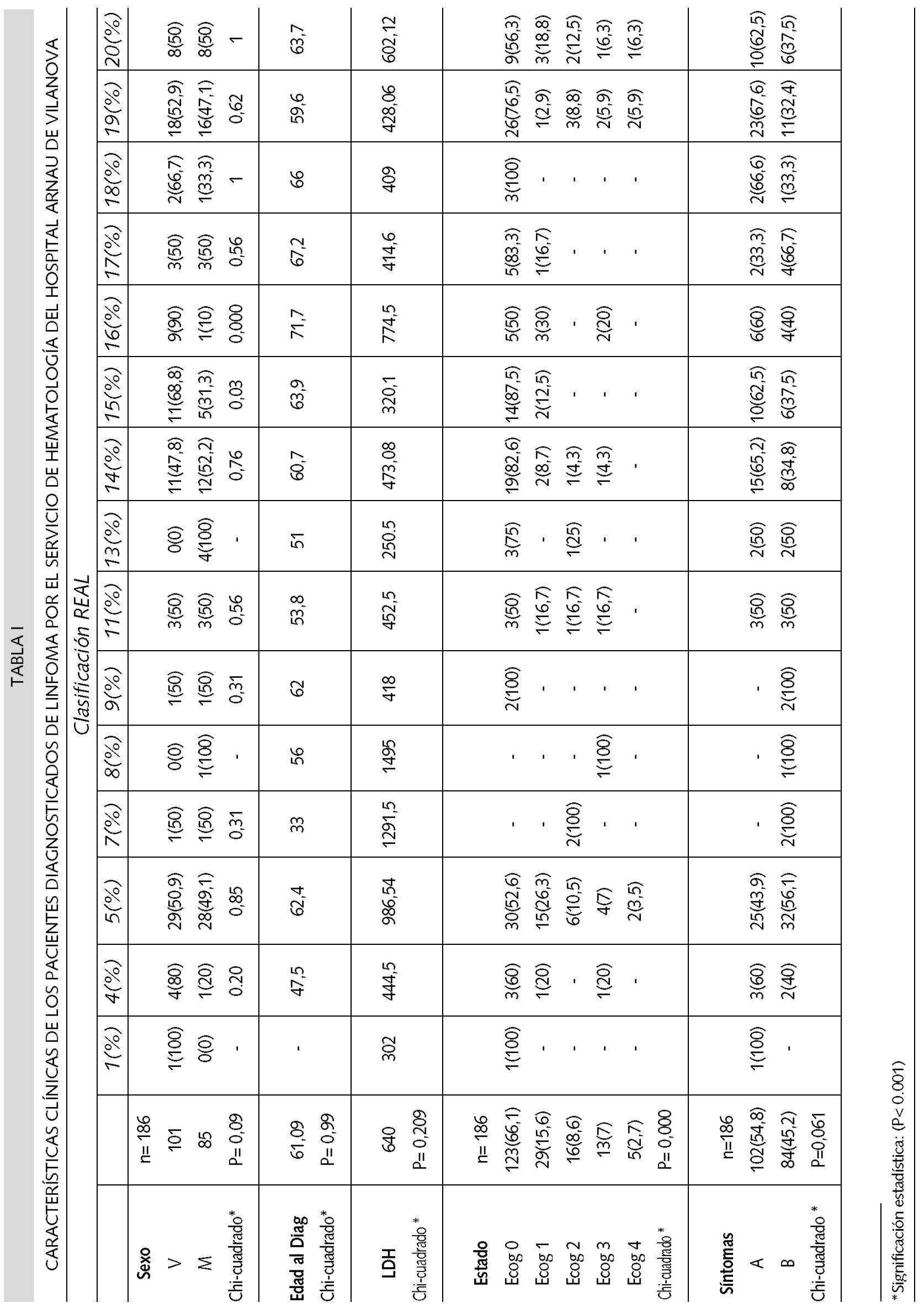




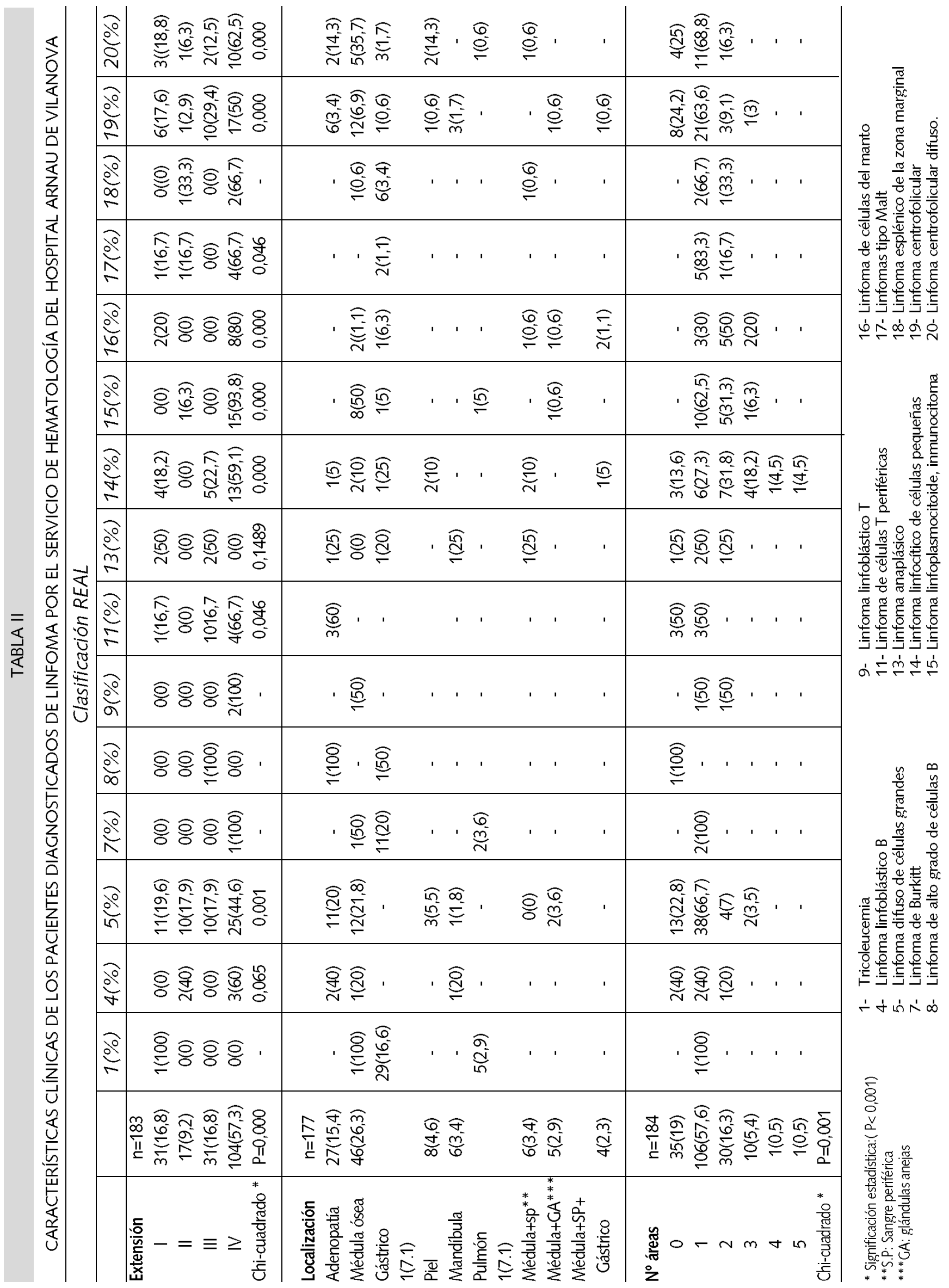


El tipo de síntomas tampoco muestra diferencias estadísticamente significativas mostrando síntomas A el 54,8\% de los casos y síntomas B el 45,2\% ( $\mathrm{p}=0,061)$.

La localización está descrita en 177 historias clínicas que suponen el 95,16\% del total de la serie. En la tabla II solamente aparecen las localizaciones con más de 4 casos.

La localización más frecuente es la de médula ósea $(26,3 \%)$ seguida de la gástrica $(16,6 \%)$ y adenopatías $(15,4 \%)$, si consideramos las ocasiones en que la localización fue médula y médula combinada con otras supone un $43,5 \%$ del total.

Por subtipos histológicos, vemos que la mayoría de los casos se presentan en el subtipo de linfoma de células grandes, siendo las localizaciones predominantes las de estómago, médula y adenopatías. Como se ve existe una gran variabilidad de la localización en este subtipo.

En cuanto al número de áreas extraganglionares afectadas en función de los diferentes subtipos diagnósticos, se observa un predominio con diferencia estadísticamente significativa $(\mathrm{p}=0,001)$ entre áreas extraganglionares afectadas y no afectadas en las que predomina el de pocas áreas afectadas, siendo en la mayoría de los casos una sola área $(57,6 \%)$. Excepto en el caso de linfoma linfocítico de células pequeñas, en que la media de áreas afectadas es de 1,86 (SD: 1,28 ) próximo a dos.

Es importante indicar que para el cálculo de los valores de significación hemos utilizado en el análisis los casos en los que aparecían más de tres pacientes, debido a la gran dispersión de los datos encontrados.

\section{DISCUSIÓN}

Los linfomas no hodgkinianos (LNH) son neoplasias de los componentes celulares del sistema inmune y de sus precursores. Si los consideramos como grupo, estos tumores tienen muchos rasgos en común, pero individualmente también reflejan la diversidad de las células normales de las que provienen, mostrando un amplio abanico de características clínicas y biológicas diferenciadas (11). Por ello, bajo el término de LNH se agrupa un conjunto de entidades nosológicas ciertamente heterogéneo con comportamiento clínicos diferenciados que hace necesaria su identificación. Es ampliamente reconocido que el factor más importante para el pronóstico de los LNH es su tipo histológico, lo que es muy importante para una correcta atención clínica y terapéutica (14)

Estas diferencias pueden ser tan acusadas que autores como DeVita aconsejaron, ya en 1975, el abandono del término LNH y su sustitución por nombres específicos de las más de 15 enfermedades en que puede dividirse (15).

Un aspecto importante a destacar es la gran dificultad intrínseca que supone el diagnóstico anatomopatológico de los LNH. Incluso entre patólogos expertos existe una sorprendente discordancia de diagnósticos, como prueban los estudios de los mayores grupos de trabajo sobre LNH (11): El Southwest Oncology Group -SWOG- (16-17), el Southeas tern Cancer Study Group (18) y el Eastern Oncology Group -ECOG- (19). En estos grupos la concordancia de diagnósticos varió ampliamente, entre el 86 y el 53\% (20). La clasificación REAL se seleccionó ya que, en muy poco tiempo, ha tenido una gran repercusión internacional y puede afirmarse que es la clasificación de referencia actual para la mayoría de los anatomopatólogos (11) .
No son bien conocidas las diferencias de incidencia de los diferentes subtipos histológicos, en parte debido a la variedad de las posibles clasificaciones y en parte porque este extremo no es recogido por la Clasificación Internacional de Enfermedades (11), Aunque estas entidades presentan diferencias e implicaciones diagnósticas y terapéuticas.

No hemos observado homogeneidad en la forma de nombrar y clasificar a los linfomas lo que se ha convertido en la mayor dificultad de estudio. Nos encontramos con más de 163 subtipos histológicos diferentes, lo que supone 8,6 diagnósticos por cada 10 pacientes. En la mayoría de estos casos no se indicaba el tipo de clasificación utilizada, por lo que un experto ha colaborado con nosotros para su conversión a la clasificación REAL. Esto ha supuesto un trabajo laborioso de recuperación de información en los archivos anatomopatológicos y muestra la necesidad de mayor relación entre patólogos y clínicos, frente a una patología en la que cada vez se manifiesta más claramente la necesidad de la identificación del subtipo histológico por presentar características clínicas diferentes.

De los diferentes subtipos en los que se divide esta clasificación, hemos encontrado una mayor frecuencia de casos pertenecientes a los tipos: linfoma difuso de células grandes, linfoma linfocítico de células pequeñas (LLC), linfoma plasmocitoide y linfoma centrofolicular (11-22).

Encontramos más frecuentes los linfomas linfoblásticos B que los T, cuando lo esperable era lo contrario.

Sólo el $15 \%$ de los enfermos presentan adenopatías en el momento del diagnóstico, posiblemente exista un sesgo de información en las historias clínicas ya que no aparecían indicadas en más casos.

Los linfomas difusos de células grandes constituyen el 30$40 \%$ de los casos de LNH en adultos, lo que llama la atención por ser menor proporción a la esperada, con una media de edad comprendida entre los 60-70 años, aunque existen casos en todas las edades (intervalo: 29-93 años), lo que indica que la edad no es un dato orientativo del diagnóstico. Los pacientes presentan masas ganglionares o extraganglionares de rápido crecimiento (11). Aparentemente hasta un $40 \%$ presentan localización extraganglionar (en nuestros pacientes en médula y digestivo).

Destaca el alto número relativo de linfomas linfocíticos de células pequeñas, linfomas linfoplasmocitoides/inmunocitomas (que son un subtipo reservado casi exclusivamente para la enfermedad de Waldenstrom y, por tanto, son muy raros) y linfomas centrofoliculares difusos, que también son de baja frecuencia. Los casos de LLCP, los enfermos suelen ser adultos mayores de 65 años (43,5\% de nuestro estudio). Las localizaciones se muestran en médula y médula más sangre periférica, pero casi por igual en otras localizaciones extraganglionares. Suelen tener transformaciones a otros tipos histológicos con mal pronóstico $(23,24)$.

Los linfomas linfoplasmocitoides son similares a los LLCP en cuanto a edad de aparición (encontramos una edad media de 60,7 para los LLCP y 63,3 para los linfomas linfoplasmocitoides).

Las localizaciones incluyen, en nuestros casos solamente la médula o ésta junto con la sangre periférica y/o gástrico, similares a otros estudios (11).

Los linfomas centrofoliculares (11-25-26) los hemos dividido en linfoma centrofolicular nodular (LCN) y linfoma centrofolicular difuso (LCD). Se sabe que afectan a adultos y a ancianos (encontramos en LCN que las edades incluyen desde 
los 41 a los 84 años con una media de 59,7 y los LCD incluyen edades desde los 51 a los 82 años con una media de 63,7 años, no existiendo diferenciación significativa en cuanto a sexo (11).

Coincidimos en la literatura en que la mayoría de los pacientes se diagnostican en estadios avanzados de la enfermedad (11) (50\% en estadio IV para los LCN y $62,5 \%$ para los LCD). La mayoría de los pacientes muestran la principal localización en adenopatías y/o médula ósea.

De todo esto se puede indicar que la edad y la edad al diagnóstico no son datos orientativos del subtipo histológico de LNH. Los pacientes se diagnostican en estadios muy tardíos, probablemente debido a su dificultad, siendo el estadio más frecuente el IV de la clasificación de Ann Arbor. Sin embargo, el estado general es bueno y la mayoría de los casos aparecen con síntomas A.

En cuanto a la localización, la más frecuente es la de médula aunque puede ir acompañada de otras localizaciones, presentándose en un $43,5 \%$ de los casos, lo que nos indica la importancia de controlar esta afectación.

En cuanto al número de áreas afectadas, vemos que la mayoría de los casos tienen una sola afectación extraganglionar, excepto en el caso de los LLCP, lo que nos lleva a que a mayor número de áreas afectadas puede ser una sospecha de este tipo de linfoma.

Nuestros datos y resultados se corresponden con otros estudios realizados en otras series (11) en los que se muestra también la complejidad de esta patología.

La valoración global de nuestros resultados muestra la importancia de la identificación del subtipo histológico por el Servicio de Anatomía patológica para el diagnóstico y la necesidad de homogeneizar estos criterios a una sola clasificación más práctica para la clínica y orientativa en la decisión terapéutica.

\section{Bibliografía}

1. Freedman DM, Zahm SH, Dosemeci M. Residential and occupational exposure to sunlight and mortality from non-Hodking's lymphoma: composite (threefold) case-control study. BMJ (Clinical Research Ed), 314 (7092): 1451-5

2. Hartge P, Devesa SS, Fraumeni JF, Jr. Hodgkin's lymphoma. In: Doll R, Fraumeni JF Jr, Muir CS, eds. Trends in cancer incidence and mortality, cancer surveys. Vol. 19/20. Plainview, NY: Cold Spring Harbor Laboratory Press, 1994; 423-53.

3. Hartge P, Devesa SS. Quantification of the impact of known risk factors on time trends in non-hodgkin's lymphoma incidence. Cancer Res 1992; 52 (Suppl.): 5566-9s.

4. Moreno MM, Fernández-Flores A, Paradela A, Rodríguez JM, Ageitos A, et al. Linfoma B rico en células T: Estudio multifactorial de cuatro casos. Sangre 1995; 40 (6): 471-7.

5. Rappaport H. Tumors of the hematopoietic system. En: Atlas of Tumor Pathology, sect III, fasc (Washimgton DC: Armed Forces Institute of Pathology 1966; 97-161

6. Gerard-Marchant R, Hamlin I, Lennert K, Rilke F, Stansfeld AG, Van Unnik JAM. Classification of malignant lymphoma. Br J Cancer 1975; 31 (suppl. II): 29-43.

7. Lennert K, Feller AC. Histopathology of Non-Hodgkin's Lymphomas (Based on the updated Kiel classificatiion) (2 ed). Berlín: SpringerVerlag, 1990.

8. The Non-Hodgkin's Lymphoma Pathologic Classification Project. National Cancer Institute sponsored study of classifications on NonHodgkin's Lymphomas: Summary and description of a working formulation for clinical usage. Cancer 1982; 49: 2112-35.

9. Harris NL, Jaffe ES, Stein H, Banks PM, Chan JKC, Clearly ML, et al. A Revised European-American Classification of Lymphoid neoplasms: A proposal from the International Lymphoma Study Group. Blood 1994; 84: 1361-92.

10. Chan JKC, Banks PM, Clearly ML, Delsol G, De Wolf Peeters C, Falini B, et al. A Revised European- American Classification of Lymphoid Neoplasms proposed by the International Lymphoma Study Group. A summary version. Am J Clin Pathol 1995; 103: 543-60.

11. Gómez Codina J. Linfomas B y T. Biología, clínica y tratamiento. Idepsa. 1997; Madrid.

12. Carbone PP, Kaplan HS, Musshoff K, Smithers DW, Tubiana M Report of the Committee on Hodgkin's disease Staging Classification. Cancer Res 1971; 31: 1860-1.

13. Rosenberg SA, Boiron M, DeVita VT Jr, Johnson RE, Lee BJ, Ultmann
JE Jr, et al. Report of the Committee on Hodgkin's disease Staging Procedures. Cancer Res 1971; 31: 1862-3.

14. Jaffe ES. Relationship of classification to biologic behavior of nonHodgkin's lymphomas. Semin Oncol 1986; 13 (Suppl. 5): 3-9.

15. DeVita VT, Canellos GP, Chabner B, Schein P, Hubbar SP, Young RC Advanced diffuse hisiocitic lymphoma, a potentially curable disease. Lancet 1975; 1: 248-50.

16. Jones SF, Butler JJ, Byrne GE, Coltman CA, Moon TE. Histopathologic review of lymphoma cases of Southwest Oncology Group. Cancer 1977; 39: 1071-6.

17. Metter GE, Nathwani BN, Burke JS, Winberg CD, Mann RB, Barcos M, et. al. Morphological subclassification of folicular lymphoma: Variability of diagnoses among hematopathologists, a collaborative study between the Repository Center and patholoy Panel for Lymphoma Clinical Studies. J Clin Oncol 1985; 3: 25-38.

18. Velez-García E, Durant J, Gams R, Bartolucci A. Results of a uniform histopathologic review system of lymphoma cases: A ten-year study from the southeastern Cancer Study Group. Cancer 1983; 52: 675-9.

19. Ezdinli EZ, Costello W, Wasser LP, Lenhard RE, Berard CW, Hartsock R, et al. Eastern Cooperative Oncology Group experience with the Rappaport classification of non-Hodgkin`s lymphomas. Cancer 1979; 43: 544-50.

20. Armitage JO, Cheson BD. Interpretation of clinical trials in diffuse large-cell lymphoma. J Clin Oncol 1988; 6: 1335-47.

21. Ries LAG, Hankey BF, Miller BA, Hartman AM, Edwards BK, eds. Cancer Statistics review, 1973-88. Bethesda: National Cancer Institute, 1991.

22. Richter MN. Generalized reticular cell sarcoma of lymph nodes associated with lymphatic leukemia. Am J Pathol 1928; 4: 285-92.

23. Brecher M, Banks P. Hodgkin's disease variant of Richter`s syndrome: Report of eigth cases. Am J Clin Pathol 1990; 93: 333-9.

24. Mann RS, Berard CW. Criteria for the cytologic subclassification of follicular lymphomas: A proposed alternative method. Hematol Oncol 1983; 1: 187-92.

25. Jaffe ES, Raffeld M, Medeiros LJ. Histophatologic subtypes of indolent lymphomas: Caricatures of the mature B-Cell System. Semin Oncol 1993; 20 (Suppl. 5): 3-30

26. Krol AD, Hermans J, Dawson L, Snijder S, Wijermans PW, KluinNelemans HC, et al. Treatment, patterns of failure, and survival of patients with Stage I nodal and extranodal non-Hodking's lymphomas, acording to data in the population-based registry of the Comprehensive Cancer Centre West. Cancer 83 (8): 1612-9. 